\title{
Françoise COLLIN, L'homme est-il devenu superflu?
}

Hannah Arendt, Paris, Odile Jacob, 1999, 332 p.

\section{Diane Lamoureux}

\section{(2) OpenEdition \\ 1 Journals}

\section{Édition électronique}

URL : https://journals.openedition.org/clio/150

DOI : $10.4000 /$ clio. 150

ISSN : 1777-5299

Éditeur

Belin

Édition imprimée

Date de publication : 1 avril 2001

Pagination : 245-248

ISBN : 2-85816-577-7

ISSN : 1252-7017

Référence électronique

Diane Lamoureux, «Françoise COLLIN, L'homme est-il devenu superflu? Hannah Arendt, Paris, Odile Jacob, 1999, 332 p. », Clio. Histoire, femmes et sociétés [En ligne], 13 | 2001, mis en ligne le 19 mars 2003, consulté le 22 avril 2022. URL : http://journals.openedition.org/clio/150 ; DOI : https://doi.org/ 10.4000/clio.150

Ce document a été généré automatiquement le 22 avril 2022.

Tous droits réservés 


\title{
Françoise COLLIN, L'homme est-il devenu superflu? Hannah Arendt, Paris, Odile Jacob, 1999, 332 p.
}

\author{
Diane Lamoureux
}

1 Dans la production industrielle qui entoure actuellement l'œuvre de Hannah Arendt en France, certains ouvrages se distinguent tant par leur qualité que par leur originalité. C'est le cas de celui de Françoise Collin qui aborde la pensée de Hannah Arendt à travers le prisme de la natalité. Ce qui intéresse F. Collin dans l'œuvre arendtienne, qui irrigue ses propres travaux depuis une quinzaine d'années, c'est la capacité de préserver l'agir en commun dans un monde durablement marqué par l'expérience totalitaire et par l'instrumentalisation des rapports humains et sociaux. Le titre fait d'ailleurs référence à ces deux données incontournables de notre époque : le génocide dans sa capacité à éliminer des pans entiers de l'humanité sous prétexte de purification et les progrès de la techno-science qui remettent en cause la place de l'humanité dans l'univers et sont susceptibles de rendre l'être humain superflu. Trois motifs me semblent devoir retenir notre attention dans cette ouvrage : le totalitarisme comme expérience incontournable du politique dans le monde contemporain, la pluralité du monde et, finalement, la natalité et la transmission.

Si l'on peut estimer, du moins dans les démocraties libérales occidentales, que l'expérience totalitaire est derrière nous, il s'agit d'un passé qui n'appartient pas totalement au passé, puisque, comme l'a bien mis en lumière $\mathrm{H}$. Arendt, le terreau duquel a surgi le monstre totalitaire est le même que celui qui a donné naissance à la démocratie libérale. Tous deux sont des horizons possibles de la modernité politique et s'éclairent mutuellement. Comprendre cela, c'est être attentive aux possibilités de résurgence de l'immonde et aussi à un ressort important de la réflexion arenditienne. Si H. Arendt s'intéresse au totalitarisme, c'est à la fois comme expérience fondatrice de sa réflexion politique et comme "excédent de tout régime " et "risque désormais incontournable d'une dissolution complète, violente ou sournoise, du monde commun » (p. 40). 
3 À cet égard, Françoise Collin a parfaitement raison de nous rappeler que H. Arendt est une penseuse qui, «à la lumière de la catastrophe totalitaire, traque au sein même des régimes démocratiques et dans la tradition occidentale, le risque de dissolution qui pèse sur le pouvoir de penser par soi-même et d'agir en commun qui fait la dignité des êtres humains et l'amour partagé du monde » (pp. 4041). Pour H. Arendt, juger et agir sont deux manifestations indissociables du souci du monde. C'est dans ce sens que F. Collin insiste sur le fait que «le monde commun (...) est un dispositif mobile qui se réinvente à chaque instant et qui tient son pouvoir de l'initiative renouvelée de chacun en confrontation avec les autres » (p. 5). La béance du mal rend nécessaire le travail proprement politique de création du monde commun, d'émergence "d'oasis de liberté ", de préservation de la pluralité comme rempart au "tout est possible» révolutionnaire ou totalitaire.

4 Si H. Arendt n'a pas, à proprement parler, réfléchi aux enjeux du féminisme, la sensibilité dont témoigne $\mathrm{F}$. Collin à la notion, chère à $\mathrm{H}$. Arendt, de pluralité ne me semble pas étrangère à l'expérience du féminisme contemporain. Depuis le début des années 1980, F. Collin a développé une réflexion à l'encontre de la mêmeté du monde féminin/féministe, au-delà de la fausse dichotomie identité/différence entre les sexes, de même qu'une conception de la démocratie qui sont toutes deux largement inspirées de la notion arendtienne de pluralité. Ces préoccupations reviennent sous un registre différent dans le présent ouvrage. Ainsi elle souligne que « le monde commun n'est pas le règne de la maîtrise mais du partage » (p. 68) et elle est sensible aux limites de la dimension déclaratoire des droits humains, dont les Juifs, pour H. Arendt, et les femmes, pour F. Collin, ont largement fait les frais. De la même façon, F. Collin insiste sur l'absence d'un point de vue totalisant sur le monde puisque celui-ci «est à la jointure d'innombrables perspectives même si toutes visent ensemble le même monde » (p. 69).

5 Si le monde commun est avant tout un monde partagé que personne ni aucun groupe ne peut s'approprier, il faudrait éviter de confondre la pluralité avec le pluralisme des partis dans nos systèmes représentatifs. Lorsque $\mathrm{H}$. Arendt parle de pluralité, elle insiste toujours sur la singularité qu'elle oppose au phénomène de la massification. Cette insistance sur la singularité, sur le « quelqu'un » ne pouvait qu'intéresser F. Collin qui a toujours mis l'accent, dans son travail de réflexion féministe, sur l'importance pour chacune de se dégager de l'assignation identitaire à la féminité pour pouvoir devenir «quelqu'une » à savoir un être capable d'interagir dans le monde parce que porteur d'un inconnu. Ce qui l'amène à mettre en lumière l'ambiguïté de la position arendtienne, tiraillée entre la conception antique de l'héroïsme et la conception chrétienne de la personne ou encore entre le dévoilement et l'initiative. «Hannah Arendt se tient ici encore entre deux conceptions du quelqu'un comme apparaissant: car le seul fait d'être un humain est déjà action en ce sens que sa présence interroge ce qui était donné et y introduit une dimension originale » (p. 130).

Une telle conception de la politique comme monde commun force à rompre avec le registre de la domination et de l'ordre venu d'en haut, pour aborder celui du pouvoir. Le pouvoir relève d'un vivre-ensemble et d'un agir en commun, ce qui pose évidemment le problème des exclusions auxquelles F. Collin accorde beaucoup plus d'importance que $\mathrm{H}$. Arendt. Cela entraîne également une réflexion sur les démocraties contemporaines qui reposent plus sur la représentation que sur l'implication. 
7 Là où la réflexion arendtienne croise le plus celle de F. Collin, c'est cependant dans l'idée de natalité. "Cette initiative incessante, cet art des commencements, qui s'origine dans le fait de la naissance, est le Contr'Un arendtien » (p. 13). Tout un chapitre est consacré au rapport entre pluralité et natalité qui constitue indubitablement la contribution la plus originale de cet ouvrage. Loin de relever de la pure facticité biologique, de s'inscrire uniquement dans le cycle du vivant, la naissance chez Arendt devient un thème philosophique. "Ce thème n'est pas accidentel mais central dans l'œuvre d'Arendt, et son "anachronie" n'est pas rétrospective mais prospective. Il a pu et peut apparaître à tort, à la lecture, comme le reliquat de la pensée traditionnelle alors qu'il annonce la nécessité d'une avancée, en pointant une impasse dans l'acquis politique moderne »(p. 188).

8 F. Collin commence par situer la problématique arendtienne de la natalité à l'intérieur des réflexions sur la vie qui peut simultanément être bios et zoê et surtout dans le cadre d'une réflexion sur la temporalité qui reprend et déplace les positions développées par Heidegger. «La natalité est aux confins de la fugue et de la durée, non pas un bastion contre la temporalité mais la temporalité incarnée : naître est temps " (p. 199). Naître se décline donc sur un double registre : celui de l'initiative et celui de la donation. "La naissance d'un nouveau-né, d'un nouveau venu, d'une nouvelle génération, est la modalité verticale du pluriel, celle qui relie des humains à d'autres humains qui ne sont pas leurs contemporains mais leurs successeurs» (p. 207). C'est dans cette perspective que F. Collin, à la suite de $\mathrm{H}$. Arendt, est amenée à interroger la démocratie contemporaine qui «s'est définie horizontalement dans l'ignorance ou l'oubli du temps» (p. 208), ce qui est exemplifié dans la notion de fraternité (ou son équivalent féministe, la sororité).

Cela permet de saisir le lien entre politique et éducation, de même que celui entre autorité et liberté, par une pensée de la transmission. Rappelant l'aphorisme de René Char, "notre héritage n'est précédé d'aucun testament " maintes fois repris par $\mathrm{H}$. Arendt, F. Collin élabore à partir de la réflexion de $\mathrm{H}$. Arendt une pensée de la transmission comme « ouverture à une nouvelle action plutôt que comme un processus d'accumulation» (p. 216). Si l'éducation est importante, c'est qu'elle doit préserver la capacité d'initiative des nouveaux venus. De la même façon, l'autorité ne consiste pas à tirer les vivants vers les morts mais à rendre possible de nouveaux commencements. $\mathrm{C}^{\prime}$ est ce qui explique la fascination de $\mathrm{H}$. Arendt pour les pères fondateurs de la démocratie américaine. "Ce que $\mathrm{H}$. Arendt admire dans la révolution américaine, qui est pour elle la seule révolution moderne, c'est qu'elle ne prétend pas à la table rase. Elle rompt, mais en rompant elle se greffe aussi sur ce qui est : elle garde mémoire. [...] Le pacte, d'autre part, engage l'avenir sans prétendre le déterminer» (p. 221). Ce sont les «many beginnings» qui, pour H. Arendt, sont porteurs du projet démocratique. $\mathrm{F}$. Collin ajoute que, si elle incluait le phénomène de la génération, cette démocratie pourrait s'avérer moins misogyne. Dans un monde désenchanté, la promesse peut tenir lieu d'espoir et le monde est toujours susceptible d'émerger au milieu des temps sombres.

10 L'ouvrage se termine par une évaluation critique de la réception de H. Arendt en France qui permet de comprendre la profonde distance qui sépare sa conception du politique de celle du républicanisme français mais qui montre également l'actualité d'une pensée qui «tente d'analyser et d'élaborer un être ensemble qui a fait l'épreuve de sa dissolution et qui en intègre la menace permanente » (p. 296). 
11 Dans ce livre, Françoise Collin nous livre donc une excellente introduction à la pensée de Hannah Arendt qui «n'est pas tant le rappel des grands principes d'organisation du politique qu'une recherche obstinée et lucide des formes dont a besoin notre temps et dont il est capable » (p. 17), en même temps qu'une réflexion personnelle sur les enjeux de notre époque, enjeux sur lesquels le féminisme a beaucoup à dire mais dont l'apport est si peu entendu. 